Monográfico: Reflujo Vesicoureteral

Arch. Esp. Urol., 61, 2 (320-322), 2008

\title{
UNA BUENA SOLUCIÓN PARA UN MAL CASO
}

Juan Rodó Salas.

Sección de Urología Pediátrica. Hospital Sant Joan de Déu. Barcelona. España.

Resumen.- Se presenta el caso de un varón afecto de reflujo, después de haber realizado un reimplante ureteral y aún después de haberle realizado un tratamiento endoscópico adicional. Al realizar una segunda cistoscopia para practicar un nuevo tratamiento endoscópico, se descubrió un orificio en el tercio medio del túnel del reimplante ureteral previo que actuaba de "shunt" y mantenía el reflujo. El paciente fue tratado exitosamente mediante la oclusión del orificio que mantenía este reflujo con una nueva inyección de "Dextranómero/Nasha".

Palabras clave: Reflujo. Tratamiento endoscópico. Reflujo vesicoureteral.
Summary.- We present the case of a child presenting vesicoureteral reflux after ureteral reimplantation and endoscopic treatment. In a second cistoscopy to repeat endoscopic treatment a new orifice in the medium third of the tunnel of the previous ureteral reimplantation was discovered. This orifice acted as a shunt and maintained reflux. The patient was treated by further occlusion of the orifice with another injection of "Dextranómero/Nasha".

Keywords: Reflux. Endoscopic treatment. Vesicoureteral reflux.

\section{INTRODUCCIÓN}

En la actualidad nadie discute la efectividad del tratamiento endoscópico del reflujo vesicoureteral primario. Las descripciones de este tratamiento en la literatura son abundantes (1-7). Pero no lo son tanto las que hacen referencia a la efectividad de este procedimiento en los casos de recidiva o aparición de un reflujo nuevo tras un reimplante ureteral previo.

El caso de un paciente afecto de reflujo persistente después de haberle sido practicado un reimplante ureteral y una primera inyección endoscópica de Deflux $®$ bajo el uréter reimplantado, motiva esta presentación.

\section{CASO CLÍNICO}

Paciente varón de 8 años de edad, afecto de vejiga neurógena no neurógena y reflujo vesicoureteral bilateral de alto grado, que fue tratado mediante nefrectomía izquierda, reimplante ureteral derecho y una vesicostomía cutánea posterior, entre los 3 y los 6 meses de edad. A los 7 años inició cateterismo intermitente, se cerró la vesicostomía y se le practicó una enterocistoplastia. En el control a los 6 meses de la intervención se apreció un reflujo nuevo, de bajo grado, que se decidió tratar endoscópicamente. Se inyectó Deflux $®$ debajo y a lo largo del túnel submucoso del Cohen previo. En el control subsiguiente persistía el reflujo, (Figura 1) y se decidió practicar una nueva punción. 


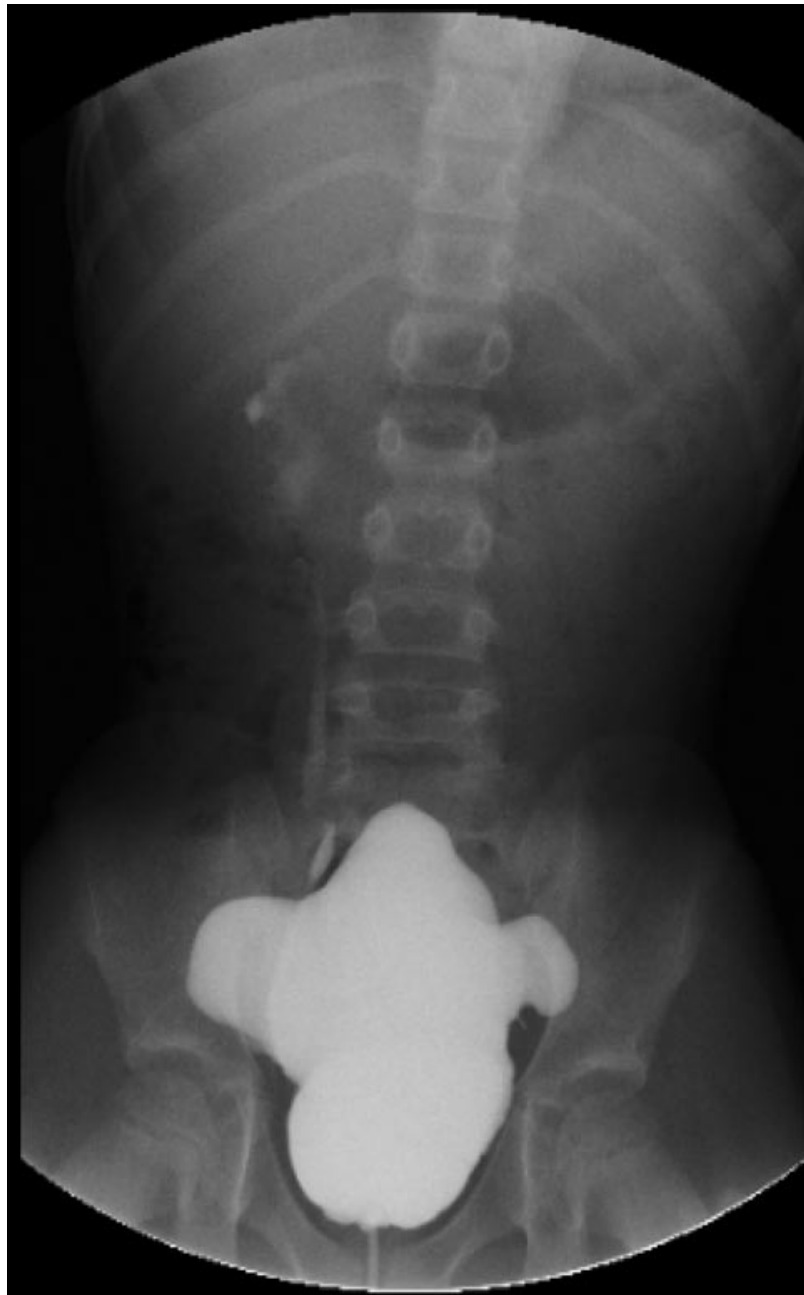

FIGURA 1. Después de un reimplante ureteral, tras el que persistía el reflujo, y después de una inyección endoscópica de Deflux ${ }^{\circledR}$, el reflujo ha disminuido de grado, pero aún persiste.

En la cistoscopia sorprendió el hallazgo de un orificio en el tercio medio del uréter que ocasionaba el reflujo (Figura 2). La punción iterativa de Deflux $®$ bajo este orificio, que actuaba como "shunt", permitió corregir el reflujo (Figura 3).

\section{DISCUSIÓN}

Las aportaciones bibliográficas que describen el tratamiento endoscópico del reflujo vesicoureteral primario con éxito, son numerosas. Se describe la técnica de la inyección (8-9) y las indicaciones, que se han ido ampliando a casos cada vez más complejos (9-10).

Cuando se trata de un reflujo persistente tras un reimplante ureteral quirúrgico, estas aportaciones son menos numerosas. Este procedimiento está descrito tras la

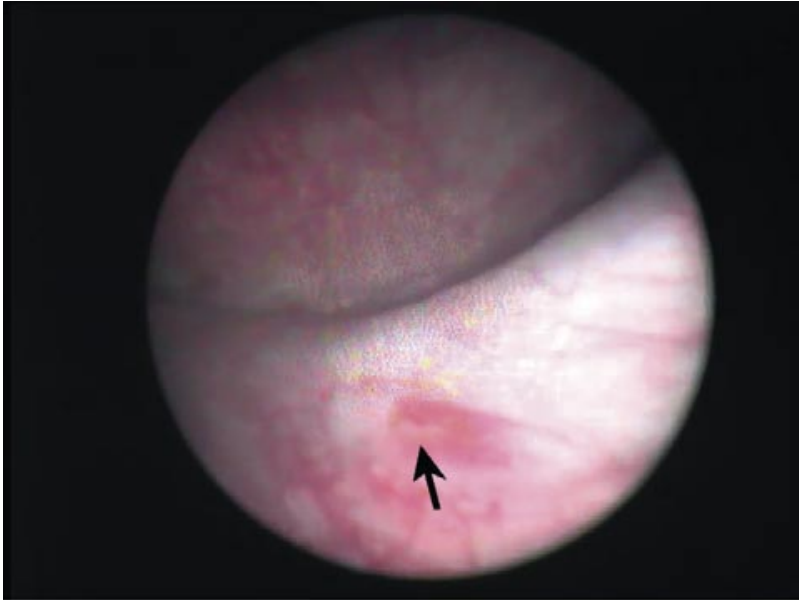

FIGURA 2. Cistoscopia. Hallazgo de un orificio en el tercio medio del uréter que actuaba como un "shunt" y ocasionaba el reflujo. Se inyectó nuevamente Deflux®, cerrando el orificio.

recidiva del reflujo después del reimplante de un uréter previamente refluyente, $(9,11)$ y tras la aparición de un reflujo nuevo después de un reimplante ureteral por megauréter no refluyente o por trasplante renal (11). Cuando con una pri-

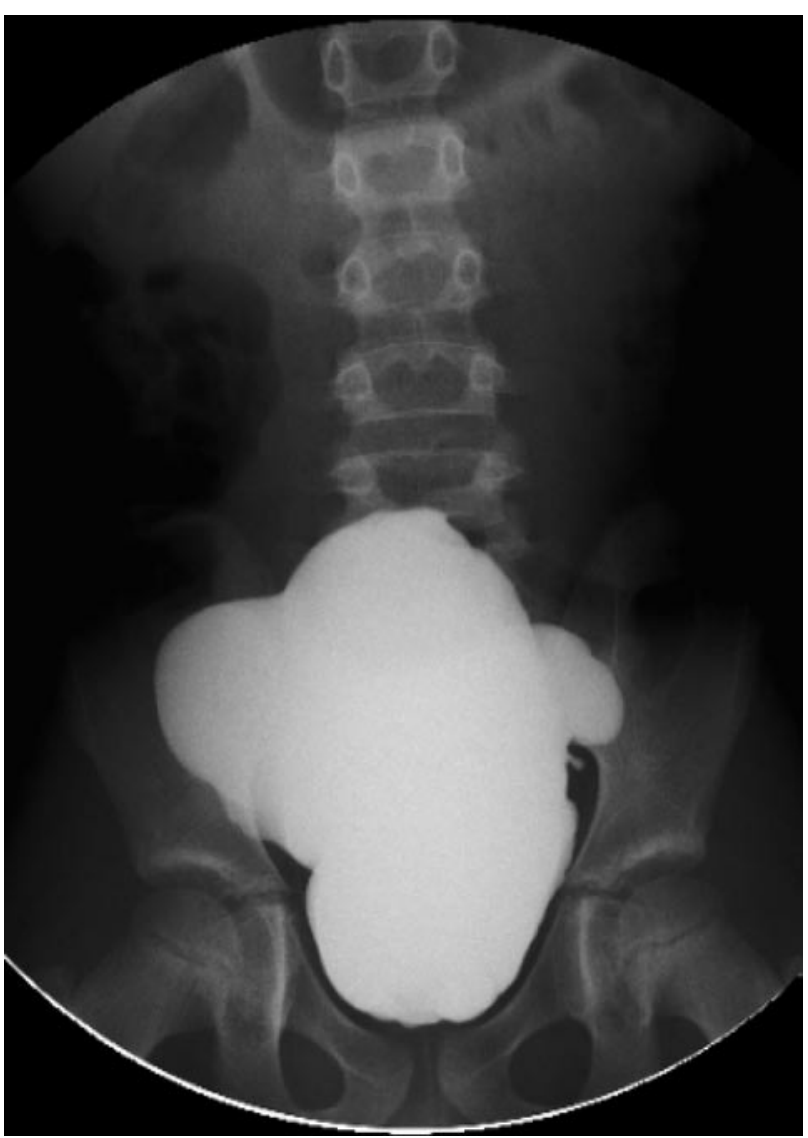

FIGURA 3. Control después de la segunda inyección. Reflujo corregido. 
mera punción endoscópica no cede el reflujo, al igual que en el reflujo primario, se pueden hacer varias punciones $y$ mejorar los resultados. (8).

No obstante puede presentarse una complicación, de la cual, hasta ahora no se ha encontrado ninguna descripción en la literatura. En nuestro caso, la causa de la recidiva del reflujo tras la punción endoscópica, fue un orificio en el túnel ureteral submucoso del reimplante previo.

Al igual que en el reflujo primario, es necesario encontrar un plano adecuado en donde depositar el material de abultamiento. El espacio virtual, que en condiciones normales, se encuentra entre la pared del uréter y el plano muscular, tras un reimplante ureteral es sustituido por una cicatriz. Si se realiza una punción a nivel de ésta, la aguja y/o el material de punción pueden penetrar en la luz del uréter, causar un decúbito, o ambas cosas. En el caso contrario, cuando la punción se realiza por debajo de la cicatriz, fácilmente se realiza el depósito del material de abultamiento por delante o por detrás del túnel ureteral submucoso y no produce el efecto deseado.

En nuestro paciente la vía utilizada para la punción fue, como habitualmente, la vía transuretral, aunque puede utilizarse la vía percutánea cuando el túnel ureteral submucoso a puncionar no es accesible (11).

\section{BIBLIOGRAFÍA y LECTURAS RECOMENDADAS ( lectura de interés $\mathrm{y}^{* *}$ lectura fundamental)}

1. PURI, P.; PIRKER, M.; MOHANNAN, N. y cols.: "Subureteral dextranomer/hyaluronic acid injection as first line treatment in the management of high grade vesicoureteral reflux". J. Urol., 176: 1856, 2006.
2. LÄCKGREN, G.; WAHLIN, N.; SKÖLDENBERG, E. y cols.: "Long-term followup of children treated with dextranomer/hyaluronic acid copolymer for vesicoureteral reflux". J. Urol., 166: 1887, 2001.

3. KOBELT, G.; CANNING, D.A.; HENSLE, T.W. y cols.: "The cost-effectiveness of endoscopic injection of dextranomer/hyaluronic acid copolymer for vesicoureteral reflux”. J. Urol., 169: 1480, 2003.

4. KIRSCH, A.J.; PEREZ-BRAYFIELD, M.; SMITH, E.A. y cols.: "The modified sting procedure to correct vesicoureteral reflux: Improved results with submucosal implantation within the intramural ureter". J. Urol., 171: 2413, 2004.

5. CAPOZZA, N.; LAIS, A.; NAPPO, S. y cols.: "The role of endoscopic treatment of vesicoureteral reflux: A 17-year experience". J. Urol., 172: 1626, 2004.

6. PURI, P.; CHERTIN, B.; VELAYUDHAM, M. y cols.: "Treatment of vesicoureteral reflux by endoscopio injection of dextranomer/hyaluronic acid copolymer: Preliminary results". J. Urol., 170: 1541, 2003.

7. STENBERG, A.; LARSSON, E.; LÄCKGREN, G.: "Endoscopic treatment with dextranomer/hyaluronic acid copolymer for vesicoureteral reflux: Histological findings". J. Urol., 169: 1109, 2003.

*8. JUNG, C.; DEMARCO, R.T.; LOWRANCE, W.T. y cols.: "Subureteral injection ox dextranomer/hyaluronic acid copolymer for persistent vesicoureteral reflux following ureteroneocystostomy". J. Urol., 177: 312, 2007.

*9. PEREZ-BRAYFIELD, M.; KIRSCH, A.J.; HENSLE, T.W. y cols.: "Endoscopic treatment with dextranomer/ Hyaluronic acid for complex cases of vesicoureteral reflux". J. Urol., 172: 1614, 2004.

10. YU, T.G.; CHANG, L.C.: "Use of collagen for endoscopic correction in complicated vesicoureteral reflux". Scand. J. Urol. Nephrol., 41: 58, 2007.

*11. KITCHENS, D.; MINEVICH, E.; DEFOOR, W. y cols.: "Endoscopic injection of dextranomer/hyaluronic acid copolymer to correct vesicoureteral reflux following failed ureterocistostomy”. J. Urol., 176: 1861, 2006. 